\title{
Human/Humanity, Consciousness and Universe: Informational Relation
}

\author{
Florin Gaiseanu*
}

\begin{abstract}
From the perspective of the Informational Model of Consciousness elaborated and reported recently on the basis of the last discoveries of the quantum mechanics and astrophysics, the meeting horizon between some ancient coherent empirical models of the humanity and our modern scientific results is analyzed. These results are discussed in terms of information, as a central axis relating the universe, the human and inter-humanity connections, and consciousness as an informational tool for the exploration of the reality. Bringing into discussion the relevant recent discoveries of quantum mechanics (Higgs' boson, disembodiment of information from the physical particles), and matter/information properties near the black holes, it is reinforced the concept of information as one of the fundamental constituent of matter and of our universe, showing that information is actually the base fabric of matter structures, living structures and universe. The huge quantity of dynamic information engaged in the living structures, particularly in the human organism, necessary to maintain the life's functions and to allow the adaptation requirements, differentiates the living from non-living entities. It is shown that consciousness, human and universe cannot be really understood if it is not introduced on the panoramic scene a new player - dark matter, with more than $20 \%$ contribution, besides more than $70 \%$ dark energy and only $5 \%$ observable matter from the matter total quantity. It is shown also that the Informational Model of Consciousness, consisting in an architecture of seven cognitive centers, converges with the ancient models of chakras, of etheric body and aura concepts, with dual Taoist concepts of universe and human body, contributing with answers to the "mind-body", "nature or nurture" problems and even to Qualia "hard" problem, and supporting the Jung's concepts on the mind. Finally, some questions are addressed to the quantum mechanics, concerning the retro-causal effect and non-locality principle.
\end{abstract}

Key Words: information, matter/dark matter, living/non-living structures, human/humanity, universe, consciousness, sentience of reality.

DOI Number: 10.14704/nq.2019.17.05.2122 NeuroQuantology 2019; 17(05):20-30

\section{Introduction}

Consciousness is a tool to explore the surrounding reality, enabling us to know ourselves and the universe. But what does it really mean "to know"? It means to gather information and to interpret it on the basis of some criteria (Gaiseanu, 2018c). Talking about ourselves, our environment and our universe, we do not yet know what the nature of this environment really is, and how it really works. Consciousness, this spectrum of knowledge that illuminates our life, is the most fascinating means by which we get to know ourselves and to try to penetrate the mysteries of nature. This continuous attempt and struggle is not new (Gaiseanu, 2019b). With thousands of years ago, the humanity tried to detect the mysteries of the human being and the universe which we live in, and he did it neither with quantum mechanics, nor with the theory of black holes, of which we are proud today, after the attainment of the first image of a black hole, an expression of our high technological level of info-creational investigation. And when we talk about „info-creational” concept, we refer not only to the powerful informational potential of our

\section{Corresponding author: Florin Gaiseanu}

Address: Principal Senior Researcher (Professor), Science and Technology of Information Bucharest (Romania) and Barcelona (Spain).

e-mail $\bowtie$ fgtext@yahoo.es.

Relevant conflicts of interest/financial disclosures: The authors declare that the research was conducted in the absence of any commercial or financial relationships that could be construed as a potential conflict of interest.

Received: 17 April 2019; Accepted: 14 May 2019 
detection tools to explore the nature mysteries, as a consequence of the high scale microelectronics and microsystem integration, but also to the huge investment of human intelligence stored in these instruments and their orientation towards a well defined goal. Our predecessors succeeded without these resources, only by means of their senses, to create empirical models (Gaiseanu, 2019b). But does this think justify to consider them irrelevant or inconclusive? Does this attitude not limits actually our own perspectives to improve our understanding on the surrounding nature? Does contemporary science comes to return today to focus on the understanding of these empirical accumulations in the light of our present outcomes? It seems that yes, this horizon is already approached (Gaiseanu, 2018d; 2019b). The informational model of consciousness recently reported in a series of articles, based on the latest discoveries of quantum mechanics (Arhanov et al, 2013) and astrophysics (Hajdukovic, 2012; 2013; Hawkins, 2005; Verlinde, 2011; Tegmark, 2014; Caroll, 2015 ) is a concrete example (Gaiseanu, 2016a,b; 2017b,c; 2018a,b,c,d; 2019a,b,c,d). With this purpose, in this paper are presented the fundamental bases of this model, starting from the concept of information of matter (Draganescu, 1979; 1990), the information in the living structures (Draganescu, 1990; Gaiseanu, 2016b), and continuing with information near the black holes ('t Hooft, 2009; Susskind, 1995) and on dark matter (Hajdukovic, 2012; 2013), a new player in our universe, which has to be taken into account to understand consciousness and mind connectivity to the environment (Gaiseanu, 2016a; $2017 \mathrm{~b}, \mathrm{c})$. A comparison with some empirical models is furthermore done, showing that our predecessors had intuitively and successfully delivered amazing results, by means of their power of sentient exploration of reality (Gaiseanu, 2019b), with which our modern and technological science could coincide. Some open questions are addressed to quantum mechanics, on the light of this informational model.

\section{Information of the universe}

In order to penetrate the still hidden properties of the human mind, let us first of all look at the still unresolved mysteries of matter and of the universe which we live in.

\section{Information of matter}

By examining ourselves, we say that we are rational beings because we think on the basis of logic. This involves however information: on one hand detection and data accumulation (Gaiseanu, 2019a), and on the other hand decision criteria, as in an informational operational system (Gaiseanu, 2018c). We create information and store it in memory on the basis of association with other existing information (Gaiseanu, 2016a). We understand today that it is one of the forms of functioning of our mind, which allows us to understand coherently the reality. We choose between Yes and No, as an informational, decisionmaking system. The balance between certainty and uncertainty marks our steps and our way to discover the new information, the goal within the scientific investigation (Gaiseanu, 2019b). That is why maybe the horizon of knowledge, depending on our own limitation, does not go too easily? That is why, everything which does not fit the limits of our own personal evidence, we ignore or consider it untrue or irrelevant? When we declare that a hypothesis is not appropriate, do we actually recognize our own ignorance? The field of knowledge is open. Nature also works with information. What we call matter today is actually a combination of matter and information. We are not still accustomed with the discovery of Higgs' boson (Anthony, 2012), which shows that mass is actually a consequence of an interaction with the Higgs' field and not an intrinsic property of matter. That is why when we talk about matter, it is no longer clear what we really have to understand by this concept: the Newtonian mass $(\mathrm{m})$, the Einsteinian energy $(E)$, coming from the relation $E=m c^{2}$ - where $c$ is the speed of the light?

A new concept enters deeper and deeper into the sciences today: information. The structuring of what we call matter is also related by information. Some decades ago, it was stated for the first time that information is a fundamental component of matter (Draganescu, 1979; 1990). What we understand today by matter, it is a consequence of a structuration process where information is directly involved (Draganescu, 1990; Gaiseanu, 2016a). Information is the one that "binds", relates the micro-components of the macrostructures. In solid bodies, the atoms are closely bonded to form a geometrically sharplydefined network, and the coherence of this structure is well built, as we detect it as a solid state. In silicon, the basic material which allowed the development of the informational systems that we benefit of today, the atoms are related by covalent bonds, actually "embodying" information. If one atom changes its state, becoming "free" to circulate through the interstitial spaces allowed by the other atoms, and 
leaving an empty site (called vacation) (Gaiseanu, 2013; 2017a), it emits information. But the atoms are not the last link of matter. To talk about matter, we need now to talk about Higgs' field and Higgs' bosons (Anthony, 2012). Eliminating layer by layer of matter, we finally come to the conclusion that our material systems, so after all the entire universe, is actually an informational system (Tegmark, 2014; Gaiseanu, 2016a; 2017b,c; Meijer, 2013a). Information is essential, but hidden fabric of matter (Gaiseanu, 2016a; Meijer, 2013a). As it was recently revealed within some quantum mechanics experiments, the properties of particles or particle systems, so information (Gaiseanu, 2016a), can be separated by the physic particles themselves (Arhanov et al., 2013). During these experiments, it was observed that the particles are "disembodied" in a separate beam from their physical body, interpreted for the first time by the physicist Gaiseanu in terms of information (Gaiseanu, 2016a, 2017b,c) and mentioned later in other articles (Meijer and Geesink, 2017; Gaiseanu, 2018d). The contribution of information to matter structuration as an intimate process was therefore definitively demonstrated (Gaiseanu, 2016a, 2017b, 2017b). The "embodiment/disembodiment" specific mechanisms of internal cell communication processes can be also defined in the same manner (Gaiseanu, 2019c).

Quantum mechanics has opened a gateway to a field of amazing properties of matter, with which we are not accustomed at the macro-structures scale. Although not understood today, only accepted as such, these properties are involved to discern the special properties of consciousness. EPR (Einstein-Podolsky-Rosen) paradox (Einstein et al., 1939; Wikipedia/EPR paradox) still preoccupies the science, through the so-called "entanglement" phenomenon. This concept has been transferred to the field of consciousness, because it allows to offer a possible explanation of the distance communication between minds (Radin, 2006), and ever more publications referring to this concept are accumulated as a scientific mainstream, although some decades ago it seemed to be a "strange" thing. Einstein himself and his collaborators called this phenomenon paradoxical, "entangling" the accounts of the erudite logical thinking. This phenomenon refers actually to quantum information, exhibiting that the exchange of information at this level between the correlated particles is done instantly, independently of separation distance. Although this phenomenon is not yet understood, it serves today to develop a new field of applications, the quantum computers, whose capacity and computing speed are greatly enhanced.

The gap between the quantum mechanics and the Newtonian mechanics was not covered yet. While in classical physics a state of a system could be firmly characterized by one of the informational states " 0 " or "1", equivalent to Yes or No in an informational decisional system, in quantum physics a particle can be found in any state as long as it is not observed, and can "collapse" in one of the states defined above. The String Theory (Wikipedia/String theory) is an attempt to cover this gap. The use now of the concepts of "superposition" and quantum "collapse" to one of "1" or " 0 " state of a particle provides hypothetical working tools that still cover our lack of certainty in this field.

The quantum experiments recently reported (Arhanov et al., 2013) decisively show that information is a detachable, distinct component of matter (Gaiseanu, 2016a; 2017b,c). Therefore, when we refer to matter, we have to understand this concept as matter-energy-information (Gaiseanu, 2016a; 2019d). Moreover, we can actually talk about an informational field of matter, containing its information, as it was defined for the first time earlier (Gaiseanu, 2016a, 2017b).

\section{Information of living structures, or matter intelligence}

By transferring this concept to the domain of consciousness, as an informational system capable of detecting, operating and generating information, it is justifiable and natural to define the infocreational field of consciousness (Gaiseanu, 2016a; $2017 \mathrm{~b}, \mathrm{c}$ ), where new information is acquired and operated or stored. The mind itself creates new information, starting from the acquired one. This field would consist in the data (information), which the mind has access to in the common sense (life experience) by the informational operator which is the thought (Gaiseanu, 2016a; 2017b), but also to the data detected as Extra-Sensorial Phenomena (ESP), which the mind can have access to under special conditions (training, specific practice, special native endowment) by direct exploration of the information field of matter (Gaiseanu, 2016a, $2017 \mathrm{~b}, \mathrm{c}$ ). In a recent publication, such a capacity was defined as Cognitive Sentient Exploration of Reality (CSER), as a tool to explore our own body, nature and universe by our sentience system (Gaiseanu, 
NeuroQuantology | May 2019| Volume 17 | Issue 05 | Page 20-30| doi: 10.14704/nq.2019.17.05.2122

Gaiseanu F., Human/Humanity, Consciousness and Universe: Informational Relation

2019b), not only in day by day activity, but also in specific creational or evaluation fields as art and science. Such a personal, subjective experience is reflected in language, in the inter-communication abilities, in the scientific or ordinary discourse, with a definite influence not limited only to the private life, but also to the social and professional fields like decisional spheres, medicine, finance, low and high education, intelligence structures (Gaiseanu, 2019b). This highlights the collective character of mind, as an "universal" global collection and connection by an informational intercommunion network. As it was also shown recently (Gaisenu, 2019c), our milliards of body cells maintains a continuous intercommunication connection in a multi-collaborative informational network supervised and managed by the brain, in order to adapt the functions of our organism as a whole to the internal and external reality, united by a common goal: survival.

The sentience is not only a specific and restrictive property of the human and humanity. From the perspective of the information concepts, one simplest unit of information Yes/No is represented by the action/reaction process, where the "sentient" side would be represented especially by the reactive participant, although the "active initiator" also experiences this interaction inside of its own system. From the informational perspective, it would result therefore that nature and universe are "sentient", its parts interacting each others and "sensing" this interaction by inside consequences. In the semiconductor technology, particularly based on silicon material, after the great development of microelectronic processors with high scale of integration, a special growing field is now dedicated to "sensing" devices, detecting not only electrical signals, but also light signals (photovoltaic and infrared cells, digital camera photo-sensitive arrays, to name only some of them), pressure capacitive sensors with biomedical application (Gaiseanu et al., 1993), piezoelectric devices and many others. Made of the basis of solid-state structures, it seems that the solid-state is also very "sentient" matter, although solid, with external unmodified properties. However, nature built already much more sophisticated "integrated-sensor devices" - the living sentient structures, starting from unicellular organisms, which seem to dispose of a "neural"- type behavior reactivity (Pereira, 2016), continuing with much more advanced sentient organisms - the animals (Proctor et al., 2013) till human, with a high level of sentience, which can be described and understood by means of the informational model of consciousness and the derived CSER tool, as it was recently shown (Gaiseanu, 2019b).

Within this context, the para-normal (PSY) capacity, so called precisely because of its out-ofcommon characteristics, explains the connection to associated phenomena, also labeled as special - the para-normal property of mind. And when we talk about matter, we refer both to matter of nonliving and living structures. But this time in the broad sense of this concept, of matter embodying information. Emphasizing the astonishing capacity of the living nature to structure and adapt through its form, architecture and specific properties, the neurologist and psychiatrist Dumitru Constantin Dulcan referred to this extraordinary acquirement as to the intelligence of matter (Dulcan, 2009). Therefore, we should not be surprised already that the living structures has been adding step by step to their own capacity of detection an even larger extrasensorial category of signals, besides of the common senses (Gaiseanu, 2019d).

But what distinguishes the living matter from the non-living matter structures? While the bulk of the solid state bodies referred above contains information which is sufficiently stable to appear unmodified at the macroscopic scale, although inside of them imperceptible atomic changes occur, the living systems contain a huge dynamic amount of information, the dynamic information quantity being majoritarian. This enormous quantity allows the reactivity to the environmental conditions and, ultimately, the adaptation to such conditions for survival. These "super"- informed structures have become increasingly "intelligent", capable of operating with information in their own benefit, intelligence becoming ultimately a predominant and representative tool for survival, proved by the informational development level of human and humanity today. Why should we therefore wonder nowadays that the human is appropriately endowed and capable to be "informed" on his environment? Just to highlight the decisive contribution of information in the structuring and functioning of living systems, particularly the human body, this was defined as "informed matter" (Gaiseanu, 2016a, 2017b,c, 2019a). This living matter informs the central decision manager (the brain), by means of the internal sensors, about its own state, allowing to regulate its proper operational system. On the 
other hand, by means of the external sensors, this also informs the brain on the changes or the state of the external environment, for optimal adaptation. Within the Informational Model of Consciousness (IMC) (Gaiseanu, 2016a; 2017b,c; 2018d; 2019c), it was stated that these two main operational activities are complied by the programmed informational system (PIS), assuring a stable maintenance of the body, and by the operative informational system (OIS) respectively, allowing the adaptive decisions.

\section{Black holes and information}

We are today the witnesses of an unprecedented and long-awaited event: the image of a black hole in our universe. It's not been too long time since Einstein guessed it within his theory of relativity (Wikipedia/ Black hole). It is perhaps again a proof of the penetrant spirit of the human mind, but of course not the only one. However as usual, some scientific disputes have also occurred in this case, solved quite recently. The need to justify the conservation of information in the universe, against the initial opinion but later revised by the famous physicist Hawkins himself (Hawkins, 2005), who predicted the emission of radiation (so information) from the black hole, led to the definition of the so-called horizon of events (Wikipedia/Event horizon), where the associated information is actually found as a holographic projection (' $t$ Hooft, 2009; Susskind, 1995). Therefore, the law of the matter conservation in universe (including information) is not violated, even under the extreme conditions of the black hole, this unimaginable concentration of mass collapsed by its own enormous gravity, able to "absorb" also the light.

Besides the purely scientific aspect of conservation of information, this dispute has clearly revealed an amazing conclusion: the information is found on the horizon of events of the black hole, which is practically a surface around it. The black hole is distinguished from the regular conditions of our around environment by its enormous mass concentrated inside, allowing therefore conclusions about the interaction between matter and information under such extreme conditions. By extrapolating, we can admit therefore that around any conglomeration of matter, that is, of material structures, one can find the information contained in the formed matter body, as projected on a surface around this body. Such a hypothesis has led to the possibility to deduce the Newton's universal attraction law, showing that the gravity would be in fact an emerging force derived from entropy, which in the universe of matter where we live in, is permanently increasing (Verlinde, 2011), according to the second law of thermodynamics. The increasing of entropy determines a time arrow oriented from the past and present to the future.

If we would refer to this discovery and to interpret it in terms of living structures, we will have the amazing revelation that the information of the human body, to customize to this living structure and consciousness, is actually distributed on a surface around it, as it was suggested recently (Gaiseanu, 2018d, 2019c).

\section{A new player on the universe scene: dark matter}

The knowledge of our science on the universe and human nature, including consciousness, may be considered perennial and satisfactory, pending on a wider theory (perhaps the string theory) that would cover the gap between quantum physics and the physics of the macro-structures. But a new player, which we have to take into account when we talk about universe and consciousness, enters in the panoramic scene of the universe: dark matter. This was proposed and introduced for the first time as a scene player with direct consequences on human consciousness and life intimate fabric by the physicist Gaiseanu (Gaiseanu, 2016a,b, 2017b,c) and it was mentioned later in other articles (Meijer and Geesink, 2017; Gaiseanu 2018a,d).

In the solid state structures, particularly in the semiconductors like silicon, the bipolar state of matter is a common, natural evidence. In the silicon lattice, the self-atoms or impurity atoms diffused inside of the bulk to modify the electrical conduction by the insertion of an excess of electrons or holes (lack of binding electrons), can be found on the positions established by the physico-mathematical order of the lattice (sites with a minimum potential energy), or in inter-spaces, as interstitial atoms, thus generating "vacancies" (empty positions after the displacement of atoms) (Gaiseanu, 2013; 2017a). The atoms and vacancies, as well as the electrons and holes, are complementary pairs with opposite properties, which could recombine, annihilating each other. If we refer to the atomic lattice, the equilibrium of such so-called punctual defects within the system is defined by thermodynamic statistical distributions. The distribution of concentration of electrons and holes is also determined by statistical laws.

Vacuum is a specific state of matter. By vacuum we do not have to understand "nothing". 
As an extreme state in nature, opposite to the black hole, it may be characterized by the fact that the mass density is extremely low. However, vacuum is actually "full" of energy and information. Einstein's simple relationship $E=m c^{2}$ shows that a mass can develop an amount of energy $(E)$. However, equally well, the relationship between mass and energy is reciprocal, the energy can generate mass. In this virtual "compact" structure of energy and information like vacuum, it is natural to think that spontaneously are generated pairs of matter and antimatter particles with opposite properties that can be also annihilated by similar recombination process as those described in the solid-state body, particularly in the depleted region (free of electric charge carriers) of a junctions (Gaiseanu et al., 1997). The life time of these particles would be very short, taking into account that matter and antimatter could explosively recombine with releasing of energy. The physicist Hajdukovic proposed a model in which these pairs become polarized due to the gravitational field where these are located, particularly the Sun and the Earth gravitational field, just as the electrical charges would be polarized in an electric field. As matter and antimatter (argued to be actually dark matter (Hajdukovic, 2012)) show anti-symmetrical properties, an immediate conclusion is that the anti-gravitational field of anti-matter would be anti-entropic, i.e. contrarily to the gravitational (entropic) field (Gaiseanu, 2016a; 2017b,c). Therefore, this field should help the life, against the entropic (destructuration) tendency of the second law of thermodynamics obeyed by matter (Gaiseanu, 2016a). A "strange" behavior would be that the time arrow in such a field is inversely oriented, from future to present (Caroll, 2015). The consciousness connection to the anti-entropic field could explain the phenomena associated with the near-death experiences (NDEs) (the return to childhood), and the premonition phenomena, which consist in a previous detection of future events (Gaiseanu, 2017b, 2017c). The consciousness connection to the field of matter (defined earlier by Gaiseanu (Gaiseanu, 2016a)) allows to the "view" intrinsic system ("eye of mind") to operate for extra-corporal explorations of reality, including the "scanning" of remote geographic regions, remote health diagnosis, and telepathic communication (Gaiseanu, 2016a, 2017b, 2018d). This is perceived in consciousness also as religious and mystic experiences (RMEs) (Gaiseanu, 2019c), inspiring peace, devotion, trust, security and confidence to humans and to entire humanity.

This hypothesis and its consequences is revolutionary, and can explain the birth of universes by successive conversion from matter to antimatter, thus eliminating the intriguing big-bang discontinuity model, the specific shape of the galaxies, as well as the expansion of the universe due to the antimatter matter-rejection process. Dark matter is majoritarian in the universe, contributing with more than $20 \%$ from total matter, besides more than $70 \%$ dark energy and only $5 \%$ observed matter. Therefore, whatever would be an explanatory model of its origin (Hajdukovic, 2013; Verlinde, 2016) its role can not be neglected or overlooked when we talk about the panoramic view of life on earth and associated properties, particularly when we refer on its influence on human, consciousness and life evolution (Gaiseanu, 2016a).

Informational Model of Consciousness and the meeting horizon with the past empirical models and with the future perspectives

The Informational Model of Consciousness - a short presentation

According to the concepts presented above, the recent proposed Information Model of Consciousness (IMC) (Gaiseanu, 2016a,b; 2017b,c,d; 2018a,b,c,d; $2019 a, b, c)$ is built on the evidence that the human body is fundamentally a bipolar structure, connected to information and to matter, so information plays a determinant role. The human thus appears as an informational structure configured by means of matter. IMC foresees the existence of seven cognitive centers corresponding to seven informational systems with fundamental functions, which represent the basic/central requirement axes of life, assuring the existence as individuals, their adaptation and the species survival: I-believe (trust, security, connection to PSY phenomena, ESP, NDEs and RMEs), I-know (internal/external capturing and storing of information - the main info-input), I-want (decisionmaking - info-output), I-love (info-emotions and emo-states (Gaiseanu, 2019c), basically representing the body's reaction to information), I-am (self-status, self-energetic power - the pole of matter connection), I-create (associativity/reproduction, genetic infoencoding - info-genetic output), I-created (inherited info-genetic-generator, species transcendental information - info-genetic input). As it was shown in a recent paper (Gaiseanu, 2019c), the captured/ memorized and repetitive information is progressively 
NeuroQuantology | May 2019| Volume 17 | Issue 05 | Page 20-30| doi: 10.14704/nq.2019.17.05.2122

Gaiseanu F., Human/Humanity, Consciousness and Universe: Informational Relation

integrated through the connectivity with the body, starting from the informational pole (low-level integration) and ending with its maximum form of info-matter integration, the genetic form. This article (Gaiseanu, 2019c), dealing actually with the mindbody problem approached from the informational perspective, shows that the traits acquired during the life by means of epigenetic mechanisms can be transmitted to descendants, demonstrating in an explicit way that human is an informational system capable not only to capture, but also to integrate the information necessary for adaptation and to transmit it as a genetic information output, without affecting the species genetic structure of the cell.

From the perspective of the above discussion, consciousness could be defined as an informational dynamic-reactive-integrative state for the sentient exploration of internal and external reality of the body, by detecting, storing, operating, processing of information and decision making, with feed-back effects toward inside and outside of the body, to adapt to the needs of the organism and to the environmental conditions, for survival. The reference to the personal "I" in the definition of the cognitive centers highlights the subjective character of consciousness, which is a personal tool of sentient exploration of reality (Gaiseanu, 2019b), supporting the philosophical concepts developed by the physician and philosopher Carl Jung (Gaiseanu, 2019b).

The meeting horizon between IMC and some past empirical models

The correlation of these centers, all of them managed by the brain, with body transducers (the main execution elements and systems), shows an obvious similitude with the architecture of the empirical model of chakras presented earlier (Ignatenko, 1994): the pole of the para-normal (PSY, EPS, NDEs) and religious experiences (RMEs) at the top of the head, the center of memory in the brain, connected with the internal and external sensors, the center of decision mainly correlated with the language system as a main form of expression, the emotional center with the heart, the self-status/power center with the digestive system, closely linked to the cardiorespirator system, and the reproduction system with the associated reproductive components (Gaiseanu, 2019a; 2019c). This similarity invites to a reflection and detailed investigation by science, and constitutes a new open gateway to a broad field of research. The resumption of this empirical system, now on scientific bases, with all its human experimental accumulation, would be of a real contribution to our today's modern neurosciences and practice of the naturist and palliative therapies (Brugnoli, 2015; Satsangi and Brugnoli, 2018).

The philosophy meets also an adequate response to its fundamental problems: "main-body" problem (mind connectivity with the body), "nature or nurture" problem (related to the inherited features expressed in IMC by the distinct specific center I-created), and even to Qualia "hard" problem (how the detected external signals are correlated with the sensations (Chalmers, 1995)).

When we talk about the projection of the body information on a surface around the body, it could seems a sterile theoretical discovery, because it is still not clear what consequences could reveal such an evidence. However, if we correlate this scientific information with some traditional models of the ancient philosophies like the chakras empirical model, we have to observe that our antecedents already knew such a near-body structure. In their empirical model, the physical body is surrounded by the so called etheric and astral body, to name only two of them (Ignatenko, 1994). In their conception, the etheric body is the most close to the physical body, assuring its health and the vital functions. According to IMC, this etheric body could be associated therefore with the information of vital functions of the organism, related to PIS. In the empiric system, the astral body, extended further away from the physical body, with lower content of information (lower "density", more "fluid"), would probably correspond to the informational activity of the info connection pole, because, according to the empirical observations, this "astral" body allows extra-corporal "travels" and geographic/astral explorations.

The empirical concept of "aura", visible by the trained persons, could be correlated with the electromagnetic activity of the brain, which today is largely used in wireless or direct commands addressed by OIS launched thoughts to the external helping motor-execution systems (Kaku, 2014). The electromagnetic activity of the brain and associated thoughts could be studied today by a non-invasive and nondestructive method, known as thefunctional MagnetoResonance Imaging (fMRI), a real emblem of our high technological level. Our predecessors, according to the local tradition transmitted and maintained today in the Tibetan philosophy, knew "read" (decipher) 
in aura, the spirit state of the person, depending on its color dynamics, like in a "video"-radiography or fMRI machine which we dispose of today, thanks to our advanced top technologies... So, although this analogy seems to be again still "strange", we should not be still surprised by the "strange" evidences revealed by the quantum mechanics and astrophysics discoveries, and we should look and boldly face such empirical evidences, as forms of challenge for new investigations. Assuming the ancient experience, this time on scientific bases, would be a beneficial advance for the human and humanity, by the useful applications which this knowledge could reveal on various field of human activities.

In the same way, we have to observe that the bipolar informational (matter/antimatter) structure - type of the universe, which IMC talk about, it was earlier deciphered by the Taoist Yin/Yang philosophy (Odoul, 2014), which is actually equivalent to the Yes/No - type informational unit related by the two distinct, contrary states. This already applicative experience shows very good results in the naturist therapies of the Chinese medicine, talking about the two form of energy (Yin/Yang) contributing/ determining the body health equilibrium. It seems therefore that this evidence is not an utopia, this was based on the sentient-intelligent detection of the cosmic reality, with corresponding consequences on everything which forms and signifies our life on our planet.

The bi-sexual reality of the human species and that of the majority species of our planet could be also a consequence of this cosmic reality, but this will be amply discussed in a future upcoming book (Gaiseanu, 2019d), which will be edited soon in various languages.

Psychokinetic phenomena can also be approached from the perspective of IMC. The mind's potential to induce psycho - mechanical effects on particles or bodies (Radin, 2018), including own body (levitation), surprises the science, which is still unprepared to respond to such challenging experiences, and the general strategy is to ignore these phenomena or to declare them as impossible. The connection to the anti-gravitational field and the probable amplification of the anti-gravity force by the power of the mind would be a "strange", but surprisingly useful hypothesis. Within this context, an evidence that can not be overlooked is the influence of the planet configuration during the period of intensive formation of the fetus nervous system, nearly the birth period, known in the popular tradition as the zodiacal set of typical properties. The reactivity of the nervous system to information over the course of the life, described by such typical common characteristics observed empirically over time, invites to reflection and systematic research (Gaiseanu, 2016a).

Immortality is a subject approached by the philosophies of many regions of the earth, and preoccupies the human and humanity (Meijer, 2013b). In the Tibetan cultures, to refer only to this concrete example, it is believed that the human beings maintain after death their informational identity, which can be "reincarnated" in a new body. The sceptics of course would reject such an hypothesis. However, during the NDEs experiences, the transit of a "tube" to an "after-life" field is typical (Gaiseanu, 2017b; 2018a). This phenomenon can be explained within IMC by the disembody of consciousness as a separate informational entity from his supporting matter ("black-hole" like system) to become a "freetype" (non - matter related) coherent informational entity. According to IMC, this process is possible because consciousness is an anti-entropic entity, helping the survival, so life), of the same (antientropic) nature like the dark matter field. Because this field is anti-entropic, then it supports also the coherence of this info-structure. Such properties should also support an (unknown) time period of its existence... If consciousness disembody is possible, then at least theoretically the "re-embodiment", the reverse process, could be possible, but this issue will be discussed in a future book (Gaiseanu, 2019d).

\section{An open horizon for future prospections}

Some debating problems addressed to the future could derive from the following questions presented below.

Asthe thoughtcanconnectand directlyinfluence the cells activity of the body, and can also connect the external environment through the informational field, could it at least influence the microparticle events? A quantum mechanic experiment (Popescu, 2013) shows that the implied particles "guess" the priory intention of the experimenter to organize a certain specific measurement. The given explanation would be that the future could influences the present (retro-causality effect in quantum mechanics experiments). However, recent works show actually a controversial, non-conclusive debate on this issue 
(Syga, 2017). According to IMC, both phenomena the influence of the anti-entropic field with a time arrow inversely oriented (from future to present) and the mind interaction between the human mind and particles, could be implied.

Could be explained the non-locality behavior of particles in quantum mechanics by taking into account rather a generation/recombination contributing mechanism of matter/anti-matter particles pair? By such a super-rapid mechanism, a matter particle recombined in a certain place, is to be generated in another place, to maintain the local equilibrium, creating the appearance of non-locality.

\section{Conclusions}

The informational model of consciousness is able to provide answers to the great questions of human and humanity, in terms of mind connectivity with the external environment and with own body, explaining both the common (normal) but also special (PSY, ESP, NDEs, RMEs) properties. For this, were taken into account and discussed some recent discoveries of quantum mechanics and astrophysics, showing that information could be separated (disembodied) from the physical support, and the universe is a bipolar matter - dark matter structure.

The connectivity with the body shows that the human is a bipolar entity, processing information and integrating it progressively on the seven informational centers, from the input non (or low) interacting form with the body matter, until the maximum integration in a genetic form. Epigenetic processes implied in such an integration can be described informationally by means of information embodiment/disembodiment mechanisms.

Consciousness is a result of the detection/ operation with information and of the informational decision-making, as a reaction to the informational connection with the external environment and with the body itself, allowing an optimal adaptation for survival. IMC shows that the disembody of consciousness is possible, and that it can be maintained out of the physical body, but for an unknown time period.

IMC also can explain the high degree capacities of our predecessors for exploration of the mysteries of our universe demonstrated by the accuracy of some of their empirical models, elaborated on the basis of their cognitive sentient exploration of reality (CSER), used as a highly sensitive tool. In the philosophic field, IMC can also offer answers to the "mind-body" problem, "nature or nurture" problem, Qualia "hard" problem, supporting Jung's philosophy on psychiatric properties of the mind.

The architecture of the cognitive centers in correlation with the executive body transducers shows a similar distribution to that of the ancient empirical chakra model. The info-surface distribution around the black holes, extrapolated to the living structures seems to support the empirical model of the "etheric", "astral" bodies and "aura" promoted by some oriental cultures.

The dual Chines philosophy on the universe, extended to the human body, with notable results in the therapy medicine can be also correlated with the bipolar structure of the universe discussed within IMC.

The popular accumulated experiences expressed by zodiacal typology of nervous reactivity is discussed in terms of the influence of the gravitational configuration during the intensive formation of the nervous system of the fetus.

From the perspective of IMC, some questions of quantum mechanics concerning the retro-causal effect and non-locality problem remain open to some alternative hypotheses.

We are still in the dawn of the great discoveries which the human and humanity begins to reveal.

\section{Acknowledgment}

To my son Adrian, with his high sentience and profoundness with which scrutinizes and understands the life and the reality.

\section{References}

Aharonov Y, Popescu S, Rohrlich D, Skrzypczyk P. Quantum Cheshire Cat. New Journal of Volume Physics 2013; 15: 1-5. http_iopscience.iop.org_1367_2630_15_11_113015_art.pdf.

Anthony S. CERN: We have found the Higgs boson 'God particle' 2012; https://www.extremetech.com/extreme/132274cern-we-have-found-the-god-particle.

Brugnoli MP, Pesce G, Pasin E, Basile MF, Tamburin S, Polati E. The role of clinical hypnosis and self-hypnosis to relief pain and anxiety in severe chronic diseases in palliative care: a 2-year long-term follow-up of treatment in a nonrandomized clinical trial, Ann Palliat Med 2015; 1-17.

Carroll S. The Arrow of Time. www.proposterousuniverse.com and From Eternity to Here, the Quest for the Ultimate Theory of Time. GB: Oneworld Publication, 2015.

Chalmers D. Facing up to the problem of consciousness". Journal of Consciousness Studies 1995; 2 (3): 200-219. 
Draganescu M. Profunzimile lumii materiale (in Romanian) - The Depths of the Material World - Bucuresti (Romania): Editura Politica, 1979.

Draganescu M. Informatia materiei (in Romanian)_Information of Matter. Bucharest (Romania): Romanian Academy, 1990.

Dulcan DC. Inteligenta Materiei (in Romanian) - (Intelligence of Matter), Romania: Ed. EIKON, 2009.

Gaiseanu F, Cobianu C, Dascalu D. Dependence of the Chemical Etching Rate and Etching Time on the Post-Implanted Diffusion Depth: Application for Membrane Achievement. Journal of the Material Science Letters 1993; 12: 1652 -1653.

Gaiseanu F, Sachelarie M, Sachelarie D, Esteve J. Analytical Modeling of the Gold Diffusion Induced Modification of the Forward Current Density Through the p-n Junctions. Solid State Phenomena 1997; 37-38: 525-530.

Gaiseanu F. Contributions to the Modelling and Simulation of the Atomic Transport Processes in Silicon and Polysilicon and Applications. PROCEEDINGS OF THE ROMANIAN ACADEMY, Series A 2013; 4(4): 376-384; www.acad.ro/sectii2002/ proceedings/doc2013-4/15-Gaiseanu.pdf.

Gaiseanu F. Consciousness as Informational System of the Human Body. Consciousness and Life Physics, Cosmology and Astrophysics Journal 2016a; 16(1): 14-25. http://physics. socionic.info/index.php/physics/article/view/227/182

Gaiseanu F. Informational Subsystems of the Consciousness. The Science of Consciousness, Abstract Book, TSC 2016 TUCSON. Ed. The University of Arizona Center for Consciousness Studies and University of Michigan Center for Consciousness Science, by Stuart Hameroff (The University of Arizona) and George Mashour (University of Michigan). 2016b. http:// www.consciousness.arizona.edu/documents/TSC2016_ B00K_of_Abstracts_for_emailWeb.pdf.

Gaiseanu F. Modeling and Simulation of the Impurity Diffusion and Related Phenomena in Silicon and Polysilicon Systems in Microfabrication and Micromachining Technologies. Annals of the Academy of Romanian Scientists, Series on Science and Technology of Information 2017a; 10(1): 41-78. http://aos. ro/wp-content/anale/IFVol10Nr1Art.4.pdf

Gaiseanu F. Quantum-Assisted Process of Disembody Under Near-Death Conditions: An Informational-Field Support Model. NeuroQuantology 2017b; 15(1): 4-9. http://www. neuroquantology.com/index.php/journal/article/view/971

Gaiseanu F. An Information Based Model of Consciousness Fully Explaining the Mind Normal/Paranormal Properties. NeuroQuantology 2017c; 15.2: 132-140. https://www. neuroquantology.com/index.php/journal/article/ view/1040

Gaiseanu F. Near-Death Experiences and Immortality from the Perspective of an Informational Modeling of Consciousness. Gerontology \& Geriatric Studies 2018a; 2(3): 1-4. https:// crimsonpublishers.com/ggs/pdf/GGS.000538.pdf

Gaiseanu F. An Informational Modeling of Consciousness and Cognitive Centers. Proc. of the Human Project Brain (HPB) International Conference: Understanding Consciousness, a Scientific Quest for the 21st Century. 04-Models, simulation and emulation of consciousness, 2018b; Barcelona: 21-22
June (2 pages): Abstract Book: http://hbp-ic.com/scientificinformation/programme/ and pdf program: http://hbp-ic. com/wp-content/uploads/2018/06/programme-hbpic2018.pdf

Gaiseanu F. Destiny or Free Will Decision? A Life Overview From the Perspective of an Informational Modeling of Consciousness Part II: Attitude and Decision Criteria, Free Will and Destiny. Gerontology \& Geriatric Studies 2018c; 4(1): 1-7. https:// crimsonpublishers.com/ggs/pdf/GGS.000576.pdf

Gaiseanu F. Information: from Philosophic to Physics Concepts for Informational Modeling of Consciousness, Philosophy Study 2018d; 8(8): 368-382. http://www.davidpublisher. org/Public/uploads/Contribute/5c06323653cd2.pdf

Gaiseanu F. Destiny or Free Will Decision? A Life Overview From the Perspective of an Informational Modeling of Consciousness Part I: Information, Consciousness and Life Cycle. Gerontology \& Geriatric Studies, 2019a; 4(1): 1-7. https://crimsonpublishers.com/ggs/pdf/GGS.000586.pdf

Gaiseanu F. Language Patterns and Cognitive-Sentient Reality: Certainty/Uncertainty in Cognitive-Sentient Exploration of Reality, Chap in Media Models to Foster Collective Human Coherence in the PSYCHecology, Ed. Stephen Brock Schafer, USA, Hershey, PA: IGI Global., 2019b :49-72. doi:10.4018/9781-5225-9065-1.ch003

Gaiseanu F. The Informational Model of Consciousness: Mechanisms of Embodiment/Disembodiment of Information, NeuroQuantology, 2019c; 17(4): 1-17. doi: 10.14704/ nq.2019.17.04.2009. https://www.neuroquantology.com/ index.php/journal/article/view/2009

Gaiseanu F. Modelere Informationala a Constiintei: De la Concepte Filozofice la Fizica Constiintei - Descoperirea Misterelor Fiintei Umane si ale Umanitătii (Romanian) Informational Modeling of Consciousness: from Philosophic Concepts to the Physics of Consciousness - Discovering the Human and Humanity Mysteries. 2019d; Globe Edit (founded in Germany 2002).

Gaiseanu F and Graur A. Cognitive Centers Related Attitude: Application for an Iterative Evaluation Method in MusicBased Therapy Process. Abstract Book of the Conference on Science of Consciousness (Co-Chair Prof. Stuart Hameroff, The University of Arizona-Center for Consciousness Studies, Tucson, Arizona). Consciousness and Education-Cognitive Development, Concurrent Session C27, 165; 2018: April 2-7. http://www.consciousness.arizona.edu/documents/ TSC2018AbstractBookfinal3.pdf

Hajdukovic D. Do we live în the universe successively dominated by matter and antimatter? Astrophys. Space Sci. 2012; 334 (2): 219-223; https://arxiv.org/pdf/1106.1260.

Hajdukovic D. Can Observations Inside the Solar System Reveal the Gravitational Properties of the Quantum Vacuum? Astrophys Space Sci. 2013; 343: 505-9.

Hawkins S. Information Loss in Black Holes. Cambridge University Press 2005; arXiv:hep-th/0507171v2.

Ignatenko A. Cum sa devii un fenomen (in Romanian)-How to Become a Phenomenon. Bucharest: Biodova, 1994.

Meijer D. Information: What Do You Mean? On the Formative Element of Our Universe. Syntropy 2013a; 3: 1-49. 
Meijer D. Immortality: Myth or Becoming Reality? On the Conservation of Information. Syntropy 2013b; 3: 168-207.

Meijer D and Geesink H. Consciousness in the Universe is Scale Invariant and Implies an Event Horizon of the Human Brain. NeuroQuantology 2017; 15(3): 41-79.

Odoul M. Ghidul complet al durerilor fizice-Terapii psihoenergetice, Prefaţă Dr. Medynski, Thierry (in Romanian, translated from Franch)-The Complete Physical Pain Guide-Psycho-Therapeutic Therapies, Preface by Dr. Medynski, Thierry. Bucuresti: Ed. Paralela 45, 2014. https:// caleaechilibruluii.files.wordpress.com/2014/04/michelodoul-ghidul-complet-al-durerilor-fizice.pdf.

Pereira C. Is it Quantum Sentience or Quantum Consciousness? A Review of Social Behaviours Observed in Primitive and PresentDay Microorganisms. NeuroQuantology 2016; 1: 16-27.

Popescu S. 'Time flow in quantum mechanics' OxfordQuantumVideo, 2013; https://m.youtube.com/ watch? $=\mathrm{cN} 3 \mathrm{akPfxfcc}$.

Proctor H, Carder G, Cornish A. Searching for Animal Sentience: A Systematic Review of the Scientific Literature. Animals 2013; 3: 882-906.

Radin D. Entangled Minds, Extrasensory Experiences in a Quantum Theory. NY, London, Toronto, Sydney: Paraview Pocket Books, a division of Simon Shuster, Inc., 2006.
Radin D. Real Magic, Ancient Wisdom, Modern Science, and a Guide to the Secret Power of the Universe. New York: Harmony Books, an imprint of the Crown Publishing Group, a division of Penguin Random House LLC, 2018.

Satsangi AK and Brugnoli MP. Anxiety and psychosomatic symptoms in palliative care: from neuro-psychobiological response to stress, to symptoms' management with clinical hypnosis and meditative states. Annals of Palliative Medicine 2018; 7(1): 75-111.

Susskind L. The World As A Hologram. J. Math. Phys. 1995; 36: 6377-6396;

arXiv:hep-th/9409089.

T Hooft G. Dimensional reduction in quantum gravity 2009; arXiv:gr-qc/9310026.

Tegmark M. Our Mathematical Universe: My Quest for the Ultimate Nature of Reality. USA: Random House, 2014.

Verlinde EP. On the Origin of Gravity and the Laws of Newton, 2011; JHEP. arXiv:1001.0785.

Verlinde EP. Emergent gravity and Dark Universe, 2016; https:// arxiv.org/abs/1611.02269.

Zyga, L. Physicists provide support for retrocausal quantum theory, in which the future influences the past. Phys Org 2017; https://phys.org/news/2017-07-physicists-retrocausalquantum-theory-future.html. 